there is only a finite number whose coefficients satisfy a relation

$$
F\left(\frac{a_{1}}{a_{n}}, \frac{a_{2}}{a_{n}}, \ldots, \frac{a_{n-1}}{a_{n}}\right)=k,
$$

where $F$ is a polynomial with positive coefficients and $k>0$; for $F$ is a polynomial in the reciprocals of the roots, and, when thus expressed, $F$ has no constant term, so that the first theorem of this paper applies. We could obtain upper bounds for the roots, and therefore for the $a$ 's, by the methods of this paper. For example, if $a_{n-1}=a_{n}$, and if $x_{1}, x_{2}, \cdots, x_{n}$ are the roots, the $x$ 's must be solutions of the equation

$$
\frac{1}{x_{1}}+\frac{1}{x_{2}}+\cdots+\frac{1}{x_{n}}=1,
$$

which has been discussed in $\S 2$.

NORTHWESTERN UNIVERSITY

\title{
ERRORS IN KRAITCHIK'S TABLE OF LINEAR FORMS
}

\author{
BY D. H. LEHMER
}

Tables of the linear forms that belong to a given quadratic residue $D$, or in other words, the linear divisors of the quadratic form $t^{2}-D u^{2}$ were first published by Legendre.* A list of errors in these fundamental tables has been given by D. N. Lehmer. $\dagger$ Kraitchik $\ddagger$ has recalculated and extended these tables to the limit $D= \pm 250$. It is of great importance in using the table that every entry be correct. Therefore in constructing his factor stencils, D. N. Lehmer found it advisable to make a new table by means of a more or less graphical method.§ This table which has not been pub-

* Théorie des Nombres, 1st. ed., Tables III-VII, 1798.

$\dagger$ This Bulletin, vol. 8 (1902), p. 401. See also the correction in this Bulletin, vol. 31 (1925), p. 228.

$\ddagger$ Théorie des Nombres, vol. 1, p. 164-186, Paris, 1922. Recherches sur la Théorie des Nombres, vol. 1, p. 205-215, Paris, 1924.

$\S$ This Bulletin, vol. 31 (1925), pp. 497-498. 
lished extends to $D= \pm 300$. The following list of errors results from collating these two tables. Kraitchik's forms for $D= \pm 182$ contain so many errors that a complete list of correct forms is given below. In as much as there were no errors detected in Lehmer's table it is reasonable to expect that the following list is complete.

Errors in Kraitchik's Table of Linear Divisors

Théorie des Nombres, vol. 1.

$\begin{array}{crr}D & \text { For } & \text { Read } \\ +38 & 59 & 53 \\ -38 & 116 & 117 \\ -42 & 55 & 53 \\ -42 & 159 & 157 \\ +69 & 55 & 53 \\ -86 & 89 & 87 \\ -102 & 147 & 145 \\ -103 & 67 & 79 \\ -103 & 177 & 179 \\ -105 & 57 & 67 \\ -106 & 73 & 71 \\ -107 & 191 & 193 \\ -109 & 333 & 103 \\ -110 & 39 & 49 \\ -110 & 207 & 217 \\ -113 & 397 & 171 \\ +122 & 195 & 199 \\ -138 & 163 & 169 \\ -141 & 413 & 415 \\ +146 & 77 & 119 \\ -146 & 77 & 303 \\ -149 & 367 & 365 \\ +151 & 183 & 189\end{array}$

Recherches, vol. 1.

$\begin{array}{ccc}D & \text { For } & \text { Read } \\ +211 & 287 & 289 \\ -217 & 319 & 317 \\ -218 & 533 & 535 \\ +222 & 99 & 95 \\ -222 & 483 & 485 \\ -226 & 375 & 373 \\ -226 & 385 & 395 \\ -226 & 387 & 397 \\ +227 & 241 & 261 \\ -229 & 197 & 199\end{array}$

Theorie des Nombres, vol. 1.

$\begin{array}{ccc}D & \text { For } & \text { Read } \\ +157 & 107 & 109 \\ -157 & 471 & 529 \\ +165 & 112 & 113 \\ -166 & 473 & 477 \\ -173 & 655 & 309 \\ +174 & 203 & 61 \\ -181 & 359 & 357 \\ -181 & 491 & 461 \\ -181 & 719 & 721 \\ -185 & 661 & 253 \\ +190 & 119 & 197 \\ +191 & 173 & 175 \\ +191 & 271 & 275 \\ +193 & 155 & 129 \\ -193 & 541 & 155 \\ -193 & 617 & 231 \\ +194 & 41 & 47 \\ -194 & 453 & 455 \\ -197 & 191 & 199 \\ +199 & 309 & 257 \\ -199 & 309 & 257 \\ -199 & \text { insert } & 371\end{array}$

Recherches, vol. 1.

$\begin{array}{crr}D & \text { For } & \text { Read } \\ +230 & 23 & 33 \\ -233 & 915 & 925 \\ -241 & 607 & 357 \\ -241 & 697 & 693 \\ -241 & 731 & 733 \\ -246 & 387 & 389 \\ -247 & 105 & 449 \\ -249 & 197 & 695 \\ -249 & 301 & 799\end{array}$


Correct Tables for $D= \pm 182$

$\begin{array}{rrrrrrr}728 n \pm & D= & +182 \\ & 1 & 9 & 15 & 19 & 25 & 33 \\ 37 & 41 & 43 & 51 & 55 & 59 \\ 61 & 69 & 71 & 73 & 81 & 83 \\ 85 & 87 & 89 & 93 & 97 & 101 \\ 103 & 107 & 109 & 113 & 115 & 121 \\ 135 & 141 & 145 & 149 & 151 & 155 \\ 157 & 159 & 171 & 173 & 179 & 181 \\ 187 & 197 & 199 & 201 & 211 & 225 \\ 227 & 233 & 235 & 237 & 239 & 241 \\ 253 & 265 & 269 & 285 & 289 & 297 \\ 307 & 311 & 317 & 319 & 333 & 335 \\ 337 & 341 & 347 & 353 & 359 & 361\end{array}$

Brown University

$$
D=-182
$$

$\begin{array}{rrrrrrr}728 n+ & 1 & 3 & 9 & 11 & 23 & 25 \\ 27 & 31 & 33 & 37 & 41 & 47 \\ 61 & 67 & 69 & 73 & 75 & 79 \\ 81 & 85 & 89 & 93 & 95 & 97 \\ 99 & 101 & 109 & 111 & 113 & 121 \\ 123 & 127 & 131 & 139 & 141 & 145 \\ 149 & 157 & 163 & 167 & 173 & 181 \\ 183 & 191 & 197 & 201 & 207 & 215 \\ 219 & 223 & 225 & 233 & 237 & 241 \\ 243 & 251 & 253 & 255 & 263 & 265 \\ 267 & 269 & 271 & 275 & 279 & 283 \\ 285 & 289 & 291 & 295 & 297 & 303 \\ 317 & 323 & 327 & 331 & 333 & 337 \\ 339 & 341 & 353 & 355 & 361 & 363 \\ 369 & 379 & 381 & 383 & 393 & 407 \\ 409 & 415 & 417 & 419 & 421 & 423 \\ 435 & 447 & 451 & 467 & 471 & 479 \\ 489 & 493 & 499 & 501 & 515 & 517 \\ 519 & 523 & 529 & 535 & 541 & 543 \\ 549 & 551 & 557 & 563 & 569 & 573 \\ 575 & 577 & 591 & 593 & 599 & 603 \\ 613 & 621 & 625 & 641 & 645 & 657 \\ 669 & 671 & 673 & 675 & 677 & 683 \\ 685 & 699 & 709 & 711 & 713 & 723\end{array}$

